\title{
SOME TOPOLOGIES ON THE SET OF LATTICE REGULAR MEASURES
}

\author{
PANAGIOTIS D. STRATIGOS \\ Long Island University \\ Brooklyn, New York 11201 \\ (Received December 20, 1989 and in revised form November 27, 1990)
}

\begin{abstract}
We consider the general setting of A.D. Alexandroff, namely, an arbitrary set $X$ and an arbitrary lattice of subsets of $X, L . \mathcal{A}(L)$ ) denotes the algebra of subsets of $X$ generated by $L$ and $M R(L)$ the set of all lattice regular, (finitely additive) measures on $\mathcal{A}(\mathcal{L})$.

First, we investigate various topologies on $M R(L)$ and on various important subsets of $M R(\mathcal{L})$, compare those topologies, and consider questions of measure repleteness whenever it is appropriate.

Then, we consider the weak topology on $M R(L)$, mainly when $L$ is $\delta$ and normal, which is the usual Alexandroff framework. This more general setting enables us to extend various results related to the special case of Tychonoff spaces, lattices of zero sets, and Baire measures, and to develop a systematic procedure for obtaining various topological measure theory results on specific subsets of $M R(L)$ in the weak topology with $\mathcal{L}$ a particular topological lattice.
\end{abstract}

Key Words and Phrases: Lattice; $\delta$ and normal lattice; separating and disjunctive lattice; lattice semiseparation. Measure; regularity, $\sigma$-smoothness, $\tau$-smoothness, and tightness of a measure; tight set of measures. Lim sup-topology; weak topology; Wallman topology; relative compactness. Repleteness, measure repleteness, and strongly measure repleteness.

1980 Mathematics Subject Classification: 28A60, 28A33, $28 C 15$.

1. INTRODUCTION. We consider the general setting of A.D. Alexandroff [2], namely, an arbitrary set $X$ and an arbitrary lattice of subsets of $X, L . \mathcal{A}(L)$ denotes the algebra of subsets of $X$ generated by $L$ and $M R(L)$ the set of all bounded, lattice regular, finitely additive measures on $\mathcal{A}(\mathcal{L})$.

First, we investigate various topologies on $M R(L)$ and on various important subsets of $M R(L)$, compare those topologies, and consider questions of measure repleteness whenever it is appropriate. It should be noted that the first topology we investigate was first considered by Blau [8] and by Kallianpur [14] on specific subsets of $M R(L)$ and by the latter in a topological framework. We thereby generalize those results and indeed we obtain Blau's main results as special cases.

Next, we consider the weak topology on $M R(L)$, mainly when $\mathcal{L}$ is $\delta$ and normal, which is the usual Alexandroff framework. This more general setting enables us to extend various results of Varadarajan [20] and to give applications to other specific topological spaces, rather than to just Tychonoff spaces, lattices of zero sets, and Baire measures, as is done by Varadarajan. Our emphasis here is to just give an example of the type of generalizations which are possible and to develop some topological measure theory results on specific subsets of $M R(L)$ in the weak topology with $L$ a particular topological lattice. We do not attempt here a systematic study of abstract Prohorov spaces, but just give an indication of the type of results which can be obtained. 
In summary, our aim is not just for generalization, but to give a systematic procedure, in a general setting, namely that of $M R(L)$, for handling any of those special settings.

We adhere to standard terminology which can be found e.g., in $[2,4,5,11,20]$, and we review some of the more important terminology and notation used throughout the paper.

Section 1. Terminology and notation.

a)Consider any set $X$ and any lattice of subsets of $X, L$. We shall always assume, without loss of generality for our purposes, that $\varnothing, X \in \mathcal{L}$. The definitions of the following terms are found in [4]: $L$ is $\delta$, separating, disjunctive, regular, normal, Lindelöf, compact, countably compact, countably paracompact.

A subset of $X, S$, is said to be $\mathcal{L}$-compact if and only if the lattice $S \cap \mathcal{L}$ is compact. The collection of $\mathcal{L}$-compact sets is denoted by $\mathcal{X}$.

For any topological space $X$, the collection of closed sets is denoted by $\mathcal{F}$, the collection of clopen sets by $C$ and the collection of Borel sets by $B$.

b)For an arbitrary function $f$, the domain of $f$ is denoted by $D_{f}$. For an arbitrary subset of $X, S$, the characteristic function of $S$ is denoted by $\kappa_{s}$. A function $f$ from $X$ to $R \cup\left\{ \pm_{\infty}\right\}$ is said to be $L$-continuous if and only if for every closed subset of $R \cup\{ \pm \infty\}, C, f^{-1}(C) \in \mathcal{L}$. The set whose general element is a function from $X$ to $R \cup\left\{{ }_{ \pm}\right\}$which is $L$-continuous and bounded is denoted by $C_{b}(L)$. The set whose general element is a zero set of $\mathcal{L}$ is denoted by $Z(L)$.

The set whose general element is the intersection of an arbitrary subset of $\mathcal{L}$ is denoted by $t \mathcal{L}$. The algebra of subsets of $X$ generated by $L$ is denoted by $q(\mathcal{L})$.

c)Consider any algebra of subsets of $X, \mathcal{A}$. A measure on $\mathcal{A}$ is defined to be a function $\mu$, from $\boldsymbol{A}$ to $R$, such that $\mu$ is finitely additive and bounded. (See [2], p. 567.) The set whose general element is a measure on $\mathcal{A}(L)$ is denoted by $M(L)$.

For an arbitrary element of $M(L), \mu$, the support of $\mu$ is defined to be $\cap\{L \in L / \mu|(L)=| \mu \mid(X)\}$ and is denoted by $S(\mu)$.

An element of $M(L), \mu$, is said to be $L$-regular if and only if for every element of $A(L), E$, for every positive number, $\varepsilon$, there exists an element of $L, L$, such that $L \subset E$ and $|\mu(E)-\mu(L)|<\varepsilon$. The set whose general element is an element of $M(L)$ which is $L$-regular is denoted by $M R(L)$. An element of $M(L), \mu$, is said to be $L$-( $\sigma$-smooth) if and only iff for every sequence in $\mathcal{A}(L),\left\langle A_{n}\right\rangle$, if $\left\langle A_{n}\right\rangle$ is decreasing and $\lim _{n} A_{n}=\varnothing$, then $\lim _{n} \mu\left(A_{n}\right)=0$. The set whose general element is an element of $M(L)$ which is $L$-( $\sigma$-smooth) is denoted by $M(\sigma, L)$. An element of $M(L), \mu$, is said to be $L-\left(\tau\right.$-smooth) if and only if for every net in $L,\left\langle L_{\alpha}\right\rangle$, if $\left\langle L_{\alpha}\right\rangle$ is decreasing and $\lim L_{\alpha}=\varnothing$, then $\lim \mu\left(L_{\alpha}\right)=0$. The set whose general element is an element of $M(L)$

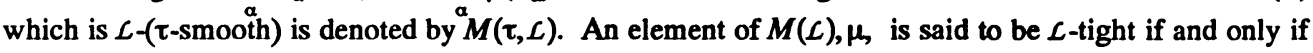
$\mu \in M(\sigma, L)$ and for every positive number, $\varepsilon$, there exists an $\mathcal{L}$-compact set, $K$, such that $|\mu| \cdot\left(K^{\prime}\right)<\varepsilon$. The set whose general element is an element of $M(L)$ which is $L$-tight is denoted by $M(t, L)$. A subset of $M(L)$, $A$, is said to be $L$-tight if and only if $A \subset M(\sigma, L)$ and $A$ is norm bounded and for every positive number, $\varepsilon$, there exists an $\mathcal{L}$-compact set, $K$, such that whenever $\mu \in A$, then $|\mu| \cdot\left(K^{\prime}\right)<\varepsilon$.

The set whose general element is an element of $M(L), \mu$, such that $\mu(\mathcal{R}(L))=\{0,1\}$ is denoted by $I(L)$. For an arbitrary element of $\mathcal{A}(L), A,\{\mu \in I R(L) / \mu(A)=1\}$ is denoted by $W(L)$ and $\{\mu \in I R(\sigma, L) / \mu(A)=1\}$ by $W_{\mathrm{o}}(\mathcal{L})$.

d) $\mathcal{L}$ is said to be replete if and only if whenever $\mu \in I R(\sigma, \mathcal{L})$, then $S(\mu) \neq \varnothing . \quad \mathcal{L}$ is said to be measure replete if and only if $M R(\sigma, L)=M R(\tau, L) . \quad L$ is said to be strongly measure replete if and only if $M R(\sigma, L)=M R(t, L)$. 


\section{Section 2.}

Consider any set $X$ and any lattice of subsets of $X, L$. Review the definition of the weak topology on $M R(L)$. Consider any element of $M R(L), \mu_{0}$. Now, consider any element of $C_{b}(\mathcal{L}), f$, and any element of $R^{+}, \varepsilon$; then, consider $\left\{\mu \in M R(L) / \int f d \mu-\int f d \mu_{0} \mid<\varepsilon\right\}$ and denote it by $N\left(\mu_{0}, f, \varepsilon\right)$. Further, consider $\left\{N\left(\mu_{0}, f, \varepsilon\right) / f \in C_{b}(L), \varepsilon \in R^{+}\right\}$and denote it by $S_{\mu_{0}}$. The following fact is well-known: There exists a topology on $M R(L), \tau$, such that $S_{\mu_{0}}$ is a subbase for the $\tau$-neighborhood system of $\mu_{0}$ and $\tau$ is unique.

Next, note the following:

a) For every net in $M R(L),\left\langle\mu_{a}\right\rangle$, for every element of $M R(L), v, \lim \mu_{a}-v$ in $\tau$ if and only if for every element of $C_{b}(\mathcal{L}), f, \lim _{\alpha} \int f d \mu_{\alpha}=\int f d v$.

b) For every element of $C_{b}(L), f$, the function $\hat{f}$ determined by $\hat{f}(\mu)=\int f d \mu, \mu \in M R(L)$ is $\tau$-continuous. Moreover, $\tau$ is the weakest topology having this property. For this reason, $\tau$ is called the weak topology on $M R(L)$.

Assume $\mathcal{L}$ is $\delta$ and normal. Then the normed vector space $M R(L)$ is isomorphic with the conjugate space of $C_{b}(L)$. (See [2], p. 577, Theorem 1.) Consequently the topology $\tau$ coincides with $\sigma\left(M R(L), C_{b}(L)\right.$ ). Denote the conjugate space of $C_{b}(L)$ by $C_{b}(L)^{-}$. Then, since $\sigma\left(C_{b}(L)^{-}, C_{b}(L)\right)$ is the weak topology on $C_{b}(L), \tau$ coincides with the weak topology on $M R(L)$.

Now, recall that the relativization of the weak topology on $I R(L)$ is the Wallman topology. (See [21].)

In the sequel, denote the relativization of the weak topology to $M^{+} R(L)$ by $w^{*}$ and the Wallman topology by $W$. Let us discard the condition " $L$ is $\delta$ and normal". We will consider other topologies on $M^{+} R(L)$, the relativization of each of which to $I R(L)$ is $W$. Also, we will discover various properties of those topologies, compare them, and investigate questions of measure repleteness whenever an opportunity arises.

\section{Section 3.}

First, consider the following statement: If $\mathcal{L}$ is $\delta$ and normal, then for every net in $M^{+} R(L),\left\langle\mu_{a}\right\rangle$, for every element of $M^{+} R(L), v, \lim _{\alpha} \mu_{\alpha}=v$ in $w^{*}$ if and only if for every element of $L, L, \varlimsup_{\alpha} \mu_{\alpha}(L) \leq v(L)$ and $\lim \mu_{\alpha}(X)=v(X)$. (See [20], p. 182, Theorem 2.) Before stating Theorem 2, Varadarajan notes that its proof is well-known and gives the following reference: ([3], p. 180, Theorem 2). However, it is to be noted that Alexandroff's theorem pertains to sequences, and its proof is rather long and not adaptable to nets. Elsewhere, the theorem mentioned in Varadarajan's paper is stated without proof or is proved in a topological setting. A proof of the statement mentioned above is given in this section.

Now, topologize $M^{+} R(L)$ as follows:

$\alpha)$ Consider any net in $M^{+} R(L),\left\langle\mu_{m}\right\rangle$, and any element of $M^{+} R(L), v .\left\langle\mu_{m}\right\rangle$ is said to converge to $v$ if and only if

(i) For every element of $L, L, \varlimsup \varlimsup_{m} \mu_{m}(L) \leq v(L)$ and

(ii) $\lim _{m} \mu_{m}(X)=v(X)$.

The statement " $\left\langle\mu_{m}\right\rangle$ converges to $v^{n}$ is also expressed as $\lim _{m} \mu_{m}=v$.

B) Define an operator on $P\left(M^{+} R(L)\right)$ as follows: Consider any element of $P\left(M^{+} R(L)\right)$, $A$. Now, consider the element of $\mathcal{P}\left(M^{+} R(L)\right), \bar{A}$, determined by $\bar{A}=\left\{v \in M^{+} R(L) /\right.$ there exists a net in $A,\left\langle\mu_{m}\right\rangle$, such that $\left.\lim _{m} \mu_{m}=v\right\}$. Show the operator "-" is a closure operator. To do this, show the Kuratowski closure conditions are satisfied. (See [15].) (Proof omitted.) Hence the operator "-" is a closure operator.

$\gamma$ ) Consider the topology on $M^{+} R(L)$ associated with this closure operator and denote it by $\mathcal{T}$. 
Observation. Consider any element of $M^{+} R(L), \mu_{0}$. Now, consider any element of $L, L$, and any element of $R^{+}, \varepsilon$; then, consider $\left\{\mu \in M^{+} R(L) / \mu\left(L^{\prime}\right)>\mu_{0}\left(L^{\prime}\right)-\varepsilon\right.$ and $\left.\left|\mu(X)-\mu_{0}(X)\right|<\varepsilon\right\}$ and denote it by $B\left(\mu_{0}, L^{\prime}, \varepsilon\right)$. Further, consider $\left\{B\left(\mu_{0}, L^{\prime}, \varepsilon\right) / L \in L, \varepsilon \in R^{+}\right\}$and denote it by $S_{m_{0}}$. The following statement is true: $S_{\mu_{0}}$ is a subbase for the $\mathcal{T}$-neighborhood system of $\mu_{0}$. (Proof omitted.) (For the $\sigma$-smooth case, see [1].)

Remark. J. H. Blau [8] works with the relativization of $\mathcal{T}$ to $M^{+} R(\sigma, L)$ and calls it the $A$-topology.

Special cases. 1. Consider the pair $\langle\operatorname{IR}(L), W(L)\rangle$ and topologize $M^{+} R(W(L))$ in accordance with the method described above and denote the resulting topology by $\hat{\tau}$.

2. Consider the pair $\langle\operatorname{IR}(L), t W(L)\rangle$ and topologize $M^{+} R(t W(L))$ in accordance with the method described above and denote the resulting topology by $\tilde{\tau}$.

3. Consider the pair $\left\langle\operatorname{IR}(\sigma, L), W_{\sigma}(L)\right\rangle$ and topologize $M^{+} R\left(W_{\sigma}(L)\right)$ in accordance with the method described above and denote the resulting topology by $\tau^{\prime}$.

Proposition 3.1. The relativization of $\mathcal{T}$ to $\operatorname{IR}(L)$ is $W$.

Proof. Consider any net in $I R(L),\left\langle\mu_{\alpha}\right\rangle$, and any element of $I R(L), v$.

a) Assume $\lim \mu_{\alpha}=v$ in $\tau$ and show $\lim \mu_{\alpha}=v$ in $W$.

Consider any member of the base for the $W$-neighborhood system of $v, W(L)^{\prime}$, such that $v \in W(L)^{\prime}$. Then $v\left(L^{\prime}\right)=1$. Since $\frac{\lim }{\alpha} \mu_{\alpha}=v$ in $\mathcal{T}, v\left(L^{\prime}\right) \leq \frac{\lim }{\alpha} \mu_{\alpha}\left(L^{\prime}\right)$. Consequently $\lim _{\alpha} \mu_{\alpha}\left(L^{\prime}\right)=1$. Hence $\left\langle\mu_{\alpha}\right\rangle$ is eventually in $W^{\prime}(L)^{\prime}$. Hence $\lim _{\alpha} \mu_{\alpha}=v$ in $W$.

b) Assume $\lim _{\alpha}^{\alpha} \mu_{\alpha}=v$ in $W$ and show $\lim _{\alpha} \mu_{\alpha}=v$ in $\mathcal{T}$.

(i) Consider any element of $L, L$, and show $v\left(L^{\prime}\right) \leq \varliminf \lim \mu_{a}\left(L^{\prime}\right)$. Consider the case: $v\left(L^{\prime}\right)=1$. Then $v \in W(L)^{\prime}$. Hence, since $\frac{\lim }{\alpha} \mu_{\alpha}=v$ in $W,\left\langle\mu_{a}\right\rangle$ is eventually in $W(L)^{\prime}$. Hence $\frac{\lim }{\alpha} \mu_{\alpha}\left(L^{\prime}\right)=1$. Consequently $v\left(L^{\prime}\right) \leq \frac{\lim }{\alpha} \mu_{\alpha}\left(L^{\prime}\right)$.

(ii) Note $\lim _{\alpha} \mu_{\alpha}(X)=v(X)$.

Consequently $\lim \mu_{\alpha}=\nu$ in $\mathcal{T}$.

Consequently the relativization of $\mathcal{T}$ to $\operatorname{IR}(\mathcal{L})$ is $W$.

Proposition 3.2. $\tau$ is $T_{1}$. (Proof omitted.)

The following three Lemmas are needed in showing that if $\mathcal{L}$ is normal, then $\mathcal{T}$ is $T_{2}$.

Lemma 3.3. If $L$ is normal, then for any element of $M^{+}(L), \mu$, for any two elements of $M^{+} R(L), v_{1}, v_{2}$ if $\mu \leq v_{1}, v_{2}$ on $\mathcal{L}$ and $\mu(X)=v_{1}(X), v_{2}(X)$, then $v_{1}=v_{2}$.

Proof. Assume $L$ is normal. Consider any element of $M^{+}(L), \mu$, and any two elements of $M^{+} R(L), v_{1}, v_{2}$ such that $\mu \leq v_{1}, v_{2}$ on $\mathcal{L}$ and $\mu(X)=v_{1}(X), v_{2}(X)$. To show $v_{1}=v_{2}$, assume the contrary. Then there exists an element of $L, A$, such that $v_{1}(A) \neq v_{2}(A)$. Consider any such $A$. Assume $v_{1}(A)<v_{2}(A)$. (Note this assumption does not affect generality.) Then, since $v_{1}, v_{2} \in M^{+}(L)$ and $v_{1}(X)=v_{2}(X)$, there exists a positive number, $\alpha$, such that $v_{1}(A)<\alpha v_{2}(X)<v_{2}(A)$. Consider any such $\alpha$. Since $v_{1}(A)<\alpha v_{2}(X)$ and $v_{1}(X)=v_{2}(X), v_{1}\left(A^{\prime}\right)>(1-\alpha) v_{1}(X)$. Hence $v_{1}\left(A^{\prime}\right)-(1-\alpha) v_{1}(X)>0$. Hence, since $v_{1} \in M^{+} R(L)$, there exists an element of $L, B$, such that $B \subset A^{\prime}$ and $v_{1}(B)>v_{1}\left(A^{\prime}\right)-\left(v_{1}\left(A^{\prime}\right)-(1-\alpha) v_{1}(X)\right)=(1-\alpha) v_{1}(X)$. Consider any such $B$. Since $B \subset A^{\prime}$ and $L$ is normal, there exist elements of $L, C, D$, such that $A \subset C^{\prime}$ and $B \subset D^{\prime}$ and $C^{\prime} \cap D^{\prime}=\varnothing$. Consider any such $C, D$. Since $C^{\prime} \cap D^{\prime}=\varnothing, C \cup D=X$. Consequently $\mu(C)+\mu(D) \geq \mu(X)$. Hence $\mu(C) \geq(1-\alpha) \mu(X)$ or $\mu(D) \geq \alpha \mu(X)$.

Consider the case: $\mu(C) \geq(1-\alpha) \mu(X)$. Then, since $\mu(X)=v_{2}(X), v_{2}(C) \geq(1-\alpha) v_{2}(X)$. Hence, since $v_{2}(A)>\alpha v_{2}(X)$ and $A \subset C^{\prime}, v_{2}(A \cup C)>v_{2}(X)$. Hence, since this statement is false, $\mu(D) \geq \alpha \mu(X)$. Then, since $\mu(X)=v_{1}(X), v_{1}(D) \geq \alpha v_{1}(X)$. Hence, since $v_{1}(B)>(1-\alpha) v_{1}(X)$ and $B \subset D^{\prime}, v_{1}(B \cup D)>v_{1}(X)$. Hence, since this statement is false, the assumption is wrong. Consequently $v_{1}-v_{2}$.

The following two lemmas are well-known. 
Lemma 3.4. Consider any real vector space $E$ and any sublinear functional on $E, p$. There exists a linear functional on $E, \psi$, such that $\psi \leq p$.

Lemma 3.5. Denote the general element of $\mathcal{A}(L)$ by $A$, and the general element of $M(L)$ by $\mu$. Consider the set whose general element is a function from $X$ to $R, f$, such that $f$ is $\mathcal{A}(L)$-simple and denote it by $S(\mathcal{A}(L))$. Consider the normed vector space $S\left(\mathcal{A}(L)\right.$ ) and denote it by $E$. Denote the conjugate space of $E$ by $E^{\text {. }}$.

There exists a function from $M(\mathcal{L})$ to $E^{\sim}, \phi$, such that $\phi(M(\mathcal{L}))=E^{\sim}$ and $\phi$ is an isomorphism. Specifically,

$\alpha)$ for every $\mu, \phi(\mu)$ is such that for every $f, \phi(\mu)(f)=\int f d \mu$;

$\beta)$ for every element of $E^{\sim}, \psi, \phi^{-1}(\psi)$ is such that for every $A, \phi^{-1}(\psi)(A)=\psi\left(\kappa_{A}\right)$.

Theorem 3.6. If $\mathcal{L}$ is normal, then $\mathcal{T}$ is $T_{2}$.

Proof. Assume $\mathcal{L}$ is normal. To show $\mathcal{T}$ is $T_{2}$, assume the contrary. Then there exists a net in $M^{+} R(\mathcal{L}),\left\langle\mu_{\alpha}\right\rangle$, such that there exist two elements of $M^{+} R(\mathcal{L}), v_{1}, v_{2}$, such that $\lim \mu_{\alpha}=v_{1}, v_{2}$ and $v_{1} \neq v_{2}$. Consider any such $\left\langle\mu_{a}\right\rangle, v_{1}, v_{2}$. Next, proceed according to the following plan: First, obtain an element of $M^{+}(\mathcal{L}), \lambda_{3}$ such that $\lambda \leq v_{1}, v_{2}$ on $\mathcal{L}$ and $\lambda(X)=v_{1}(X), v_{2}(X)$. Then, show $v_{1}=v_{2}$, thus reaching a contradiction.

For every $\alpha$, consider $\phi\left(\mu_{\alpha}\right)$ (see Lemma 3.5) and denote it by $\phi_{\alpha}$. consider the function $p$ on $E$, such that $p(f)=\lim _{\alpha}(f)$. Note $p$ is a sublinear functional. Hence, by Lemma 3.4, there exists a linear functional on $E, \psi$, such that $\psi \leq p$. Consider any such $\psi$.

Show $\psi$ is bounded. Note for every $f, \psi(f) \leq p(f)=\varlimsup_{\alpha} \phi_{\alpha}(f) \leq \varlimsup_{\alpha}\left|\phi_{\alpha}(f)\right|=\varlimsup_{\alpha}\left|\int f d \mu_{\alpha}\right| \leq \varlimsup_{\alpha}$ $\left(\|f\|\left\|\mu_{\alpha}\right\|\right)=\left(\varlimsup_{\alpha}\left\|\mu_{\alpha}\right\|\right)\|f\|=\left(\varlimsup_{\alpha} \mu_{\alpha}(X)\right)\|f\| . \quad$ Since $\lim _{\alpha} \mu_{\alpha}=v_{1}, \lim _{\alpha} \mu_{\alpha}(X)-v_{1}(X)$. Hence $\varlimsup_{\alpha}^{\alpha}$ $\mu_{\mathrm{a}}(X)=v_{1}(X)$. Consequently for every $f, \psi(f) \leq v_{1}(X)\|f\|$. Moreover, for every $f, \psi(-f) \leq p(-f)=$ $\varlimsup_{\alpha} \phi_{\alpha}(-f) \leq \varlimsup_{\alpha}\left|\phi_{\alpha}(-f)\right| \leq v_{1}(X)\|f\|$. Hence $\psi$ is bounded.

Now, consider $\phi^{-1}(\psi)$ (see Lemma 3.5) and denote it by $\lambda$.

Show $\lambda \in M^{+}(L)$. Note for every $A, \lambda(A)=\phi^{-1}(\psi)(A)=\psi\left(\kappa_{A}\right)$. Next, show $\psi$ is a generalized Banach limit. Show for every $f, \lim _{\alpha} \phi_{a}(f) \leq \psi(f) \leq \varlimsup_{\alpha} \phi_{a}(f)$. Note for every $f, \psi(f) \leq \varlimsup_{\alpha} \phi_{\alpha}(f)$; to show $\frac{\lim _{\alpha}}{\alpha} \phi_{a}(f) \leq$ $\psi(f)$, use the fact: $\frac{\lim }{\alpha} \phi_{\alpha}(f)=-\varlimsup_{\alpha}\left(-\phi_{\alpha}(f)\right)$. Fix $f$. Note $\psi(-f) \leq \varlimsup_{\alpha} \phi_{\alpha}(-f)=\varlimsup_{\alpha}\left(-\phi_{\alpha}(f)\right)$. Consequently $\psi(f)=-\psi(-f) \geq-\varlimsup_{\alpha}\left(-\phi_{\alpha}(f)\right)=\frac{\lim }{\alpha} \phi_{\alpha}(f)$. Consequently for every $f, \frac{\lim _{\alpha}}{\phi_{\alpha}}(f) \leq \psi(f) \leq \varlimsup_{\alpha} \phi_{a}(f)$. Hence $\psi$ is a generalized Banach limit. Now, note for every $A, \frac{\lim }{\alpha} \phi_{\alpha}\left(\kappa_{A}\right) \leq \psi\left(\kappa_{A}\right)$. Consequently for every $A$, $\lambda(A) \geq \frac{\lim }{\alpha} \phi_{a}\left(\kappa_{A}\right)=\frac{\lim }{\alpha} \mu_{\alpha}(A) \geq 0$. Hence $\lambda \in M^{+}(L)$.

Next, show $\lambda \leq v_{1}, v_{2}$ on $\mathcal{L}$. Note for every element of $L, L, \lambda(L)=\psi\left(\kappa_{L}\right) \leq p\left(\kappa_{L}\right)=\varlimsup_{\alpha} \phi_{u}\left(\kappa_{L}\right)=\varlimsup_{\alpha}$ $\mu_{\alpha}(L)$. Since $\lim _{\alpha} \mu_{\alpha}=v_{1}, v_{2}$, for every element of $L, L, \varlimsup_{\alpha} \mu_{\alpha}(L) \leq v_{1}(L), v_{2}(L)$. Consequently $\lambda \leq v_{1}, v_{2}$ on $\mathcal{L}$.

Finally, show $\lambda(X)=v_{1}(X), v_{2}(X)$. Note $\lambda(X)=\psi\left(\kappa_{X}\right)$ and $\frac{\lim }{\alpha} \phi_{\alpha}\left(\kappa_{X}\right) \leq \psi\left(\kappa_{X}\right) \leq \prod_{\alpha} \phi_{\alpha}\left(\kappa_{X}\right)$. Since $\lim _{\alpha} \mu_{\alpha}=v_{1}, v_{2}, \lim _{\alpha} \mu_{\alpha}(X)=v_{1}(X), v_{2}(X)$. Consequently $\lambda(X)=v_{1}(X), v_{2}(X)$.

Summarizing: $\lambda \in M^{+}(L)$ and $v_{1}, v_{2} \in M^{+} R(L)$ and $\lambda \leq v_{1}, v_{2}$ on $L$ and $\lambda(X)=v_{1}(X), v_{2}(X)$.

Then, since $\mathcal{L}$ is normal, by Lemma 3.3, $v_{1}=v_{2}$. Thus a contradiction has been reached. Consequently $\tau$ is $T_{2}$.

Corollary 3.7. If $\mathcal{L}$ is normal, then $I R(L)$ is closed.

Proof. Assume $L$ is normal. Recall that the relativization of $\mathcal{T}$ to $\operatorname{IR}(L)$ is $W$ and $\operatorname{IR}(L)$ is $W$-compact. Since $L$ is normal, by Theorem 3.6, $\tau$ is $T_{2}$. Consequently $I R(L)$ is closed.

Examples. (1). Consider any topological space $X$ such that $X$ is normal and let $\mathcal{L}=\mathcal{F}$. Then $\operatorname{IR}(\mathcal{F})$ is closed. 
$\operatorname{IR}(\mathcal{F})$ is known as the Wallman compactification of $X$ and is denoted by $\omega X$. (See [21].)

(2). Consider any topological space $X$ such that $X$ is $T_{3 \frac{1}{2}}$ and let $L=Z$. Then $I R(Z)$ is closed.

$\operatorname{IR}(Z)$ is known as the Stone-Cech compactification of $X$ and is denoted by $\beta X$. (See [10].)

(3). Consider any topological space $X$ such that $X$ is $T_{1}$ and 0 -dimensional and let $L=C$. Then, $I R(C)$ is closed.

$I R(C)$ is known as the Banaschewski compactification of $X$ and is denoted by $\beta_{0} X$. (See [7].)

Corollary 3.8. If $\mathcal{L}$ is normal, then $\mathcal{X} \cup\left\{M^{+} R(L)\right\}$, where $\mathcal{X}$ is the collection of $\mathcal{T}$-compact sets, is a lattice and is measure replete.

Proof. Assume $\mathcal{L}$ is normal. Then $\mathcal{T}$ is $T_{2}$. Hence, since every element of $\mathcal{X}$ is closed, $\mathcal{X} \cup\left\{M^{+} R(L)\right\}$ is a lattice and is compact. Consequently $\mathcal{X} \cup\left\{M^{+} R(L)\right\}$ is measure replete.

Proposition 3.9. If $L$ is $\delta$ and normal, then $M^{+} R(L)$ is closed.

Proof. Assume $L$ is $\delta$ and normal. Consider any net in $M^{+} R(L),\left\langle\mu_{a}\right\rangle$, and any element of $M R(L), v$, such that $\lim \mu_{a}=v$. Show $v \in M^{+} R(L)$. Since $v \in M R(L)$, is suffices to show for every element of $L, L, v(L) \geq 0$. Assume there exists an element of $L, L$, such that $v(L)<0$. Consider any such $L$. Then, by Alexandroff's Representation Theorem, ([2], p. 577, Theorem 1), $v(L)=\lim _{D(L)} \int f d v$. Hence for every positive number, $\varepsilon$, there exists an element of $C_{b}(L), f_{0}$, such that $f_{0} \geq \kappa_{L}$ and for every element of $C_{b}(L), f$, if $f \geq \kappa_{L}$ and $f \leq f_{0}$, then $\left|v(L)-\int f d v\right|<\varepsilon$. Let $\varepsilon=-\frac{1}{2} v(L)$ and consider any element of $C_{b}(L), f_{0}$, such that $-\cdots$. Then $\left|v(L)-\int f_{0} d v\right|<-\frac{1}{2} v(L)$. Consequently $\int f_{0} d v<\frac{1}{2} v(L)<0$. Since $\lim _{\alpha} \mu_{\alpha}=v, \lim _{\alpha} \int f_{0} d \mu_{\alpha}=\int f_{0} d v$. Hence, since for every $\alpha, \int f_{0} d \mu_{\alpha} \geq 0, \int f_{0} d v \geq 0$. Thus a contradiction has been reached. Consequently $v \in M^{+} R(L)$. Hence $M^{+} R(L)$ is closed.

Proposition 3.10. If $\mathcal{L}$ is $\delta$ and normal, then $\mathcal{F}$, the collection of $w^{*}$-closed subsets of $M R(\mathcal{L})$, is measure replete.

Proof. Assume $L$ is $\delta$ and normal. Then, since every norm bounded subset of $M R(L)$ is $w^{*}$-compact, $\left\langle M R(L), w^{*}\right\rangle$ is $\sigma$-compact. Hence $\left\langle M R(L), w^{*}\right\rangle$ is Lindelöf; otherwise stated: $\mathcal{F}$ is Lindelöf. Consequently $\mathcal{F}$ is measure replete.

Corollary 3.11. If $L$ is countably compact, $\delta$, and normal, then $\left\langle M R(\sigma, L), w^{\circ}\right\rangle$ is Lindelöf. (Proof omitted.)

Examples. (1). Consider any topological space $X$ such that $X$ is countably compact and normal, and let $\mathcal{L}=\mathcal{F}$. Then $\left\langle M R(\sigma, \mathcal{F}), w^{*}\right\rangle$ is Lindelöf.

(2). Consider any topological space $X$ such that $X$ is pseudocompact and $T_{3 \frac{1}{2}}$, and let $\mathcal{L}=Z$. Then $\left\langle M R(\sigma, Z), w^{*}\right\rangle$ is Lindelöf.

Proposition 3.12. If $\mathcal{L}$ is (separating) and disjunctive, then $M^{+} R(L) \subset \overline{[X]}$, where $[X]$ is the vector subspace of $M R(\mathcal{L})$ spanned by $X$. (An explanation of why the word separating is enclosed within parentheses is found in ([4], p. 1502)).

Proof. Assume $L$ is (separating) and disjunctive. Consider any element of $M^{+} R(L), v$. Note to show $v \in \overline{[X]}$, by the definition of "-", it suffices to show there exists a net in $[X]\left\langle\mu_{a}\right\rangle$, such that $\lim _{\alpha} \mu_{\alpha}=v$. Such a net is obtained as follows:

Consider any finite partition of $X$ (relative to $\mathcal{A}(L)), P$. Set $P=\left\{A_{k} ; k=1, \ldots, n\right\}$. For each $k$, consider any element of $A_{k}, x_{k}$. Then, consider $\sum_{k} v\left(A_{k}\right) \mu_{x_{k}}$. Since $v \in M^{+} R(L)$ and $L$ is disjunctive, $\sum_{k} v\left(A_{k}\right) \mu_{x_{k}}$ $\in M^{+} R(L)$. Set $\sum_{k} v\left(A_{k}\right) \mu_{x_{k}}=\mu_{p}$. Next, consider the set whose general element is $P$ and denote it by $P$. Direct $P$ as follows: for any two elements of $P, P_{1}, P_{2}$, set $P_{2} \geq P_{1}$ if and only if $P_{2}$ is a refinement 
of $P_{1}$. Now, consider $\left\langle\mu_{p} ; P \in P\right\rangle$. (The idea of constructing such a net is due to Varadarajan [20].) Show $\lim _{p} \mu_{p}=v$. (Proof omitted.) Hence $v \in \overline{[X]}$. Hence $M^{+} R(L) \subset \overline{[X]}$.

The following theorem settles the question of coincidence of the topology $\tau$ and the topology $w^{*}$ (when $\mathcal{L}$ is $\delta$ and normal), raised at the beginning of this section, and also generalizes an important result of Blau ([8], p. 27, obtained by combining Theorems 4, 5, and 6).

Theorem 3.13. a) For every net in $M^{+} R(L),\left\langle\mu_{a}\right\rangle$, for every element of $M^{+} R(L), v$, if $\lim _{\alpha} \mu_{\alpha}=v$ in $T$, then $\lim \mu_{\alpha}=v$ in $\tau$.

b) The collection of $\tau$-closed sets is contained in the collection of $\tau$-closed sets.

c) If $\mathcal{L}$ is $\delta$ and normal, then for every net in $M^{+} R(L),\left\langle\mu_{a}\right\rangle$, for every element of $M^{+} R(L), v$, if $\lim _{a} \mu_{a}=v$ in $w^{*}$, then $\lim \mu_{\alpha}=v$ in $\mathcal{T}$.

d) If $L$ is $\delta$ and normal, then $\tau$ coincides with $w^{*}$.

Outline of a proof. a) Consider any net in $M^{+} R(L),\left\langle\mu_{a}\right\rangle$, and any element of $M^{+} R(L), v$, such that $\lim _{\alpha} \mu_{\alpha}=v$ in $\mathcal{T}$. Note to show $\lim _{\alpha} \mu_{\alpha}=\nu$ in $\tau$, by the definition of $\tau$, it suffices to show for every element of $C_{b}(L), f, \lim _{\alpha} \int f d \mu_{\alpha}=\int f d v$.

Assume for every element of $C_{b}^{+}(L), h$, if $h<1$, then $\lim \int h d \mu_{\alpha}-\int h d v$. Then for every element of $C_{b}(\mathcal{L}), f, \lim _{\alpha} \int f d \mu_{\alpha}=\int f d v$. Therefore it suffices to show for every element of $C_{b}^{+}(L), h$, if $h<1$, then $\lim _{\alpha} \int h d \mu_{\alpha}^{\alpha}=\int h d v$.

Consider any element of $C_{b}^{+}(L), h$, such that $h<1$. Show $\varlimsup_{\alpha} \int h d \mu_{\alpha} \leq \int h d v \leq \frac{\lim }{\alpha} \int h d \mu_{\alpha}$. (Proof omitted.)

b) (Use a).)

c) Assume $\mathcal{L}$ is $\delta$ and normal. Consider any net in $M^{+} R(L),\left\langle\mu_{a}\right\rangle$, and any element of $M^{+} R(L), v$, such that $\lim _{\alpha} \mu_{\alpha}=v$ in $w^{*}$. (Recall $w^{*}$ coincides with $\tau$.) Show $\lim _{\alpha} \mu_{\alpha}=v$ in $\tau$.

(i) Consider any element of $L, L$. Show $\overline{\lim } \mu_{a}(L) \leq v(L)$. Since $v \in M^{+} R(L)$, there exists an element of $\mathcal{L}, L$, such that $\tilde{L}^{\prime} \supset L$ and $v\left(\tilde{L}^{\prime}\right)<v(L)+\varepsilon$. Consider any such $L$. Since $L \subset L^{\prime}$ and $L$ is $\delta$ and normal, there exists an element of $C_{b}(L)$, $f$, such that $f(L)=\{1\}$ and $f(\tilde{L}) \subset\{0\}$ and $0 \leq f \leq 1$. Consider any such $f$. Now, note for every $\alpha, \mu_{\alpha}(L)=\int f d \mu_{\alpha} \leq \int f d \mu_{\alpha}$. Hence $\varlimsup_{\alpha} \mu_{\alpha}(L) \leq \varlimsup_{\alpha} \in f d \mu_{\alpha}$. Since $\lim _{\alpha} \mu_{\alpha}-v$ in $w^{*}, \lim _{\alpha} \int f d \mu_{\alpha}=\int f d v$. Consequently $\varlimsup_{\alpha} \mu_{\alpha}(L) \leq \int f d v-\int_{\tilde{L}^{\prime}} f d v \leq v\left(\tilde{L}^{\prime}\right)<v(L)+\varepsilon$. Hence $\varlimsup_{\alpha} \mu_{\alpha}(L) \leq v(L)$.

(ii) Show $\lim _{\alpha} \mu_{\alpha}(X)=v(X)$. Since $\lim _{\alpha} \mu_{\alpha}=v$ in $w^{*}, \lim _{\alpha} \int 1 d \mu_{\alpha}-\int 1 d v$. Hence $\lim _{\alpha}^{\alpha} \mu_{\alpha}(X)-v(X)$.

Consequently $\lim \mu_{\alpha}=v$ in $\mathcal{T}$.

d) (Use a) and c).)

Corollary 3.14. If $\mathcal{L}$ is countably compact, $\delta$ and normal, then for every nonnegative number, $k$, the subspace $\left\{\mu \in M^{+} R(\sigma, L) \mid \mu(X)=k\right\}$ is $T_{2}$ and compact. (Proof omitted.)

This corollary generalizes another important result of Blau ([8], p. 31, Theorem 11).

Examples. (1). Consider any topological space $X$ such that $X$ is countably compact and normal, and let $\mathcal{L}=\mathcal{F}$. Then for every nonnegative number, $k$, the subspace $\left\{\mu \in M^{+} R(\sigma, \mathcal{F}) \mid \mu(X)=k\right\}$ is $T_{2}$ and compact.

(2). Consider any topological space $X$ such that $X$ is pseudocompact and $T_{3 \frac{1}{2}}$, and let $L=Z$. Then for every nonnegative number, $k$, the subspace $\left\{\mu \in M^{+} R(\sigma, Z) \mid \mu(X)-k\right\}$ is $T_{2}$ and compact.

The following theorem generalizes a result of Kallianpur ([14], p. 948, Theorem 2.1).

Theorem 3.15. Consider the condition: For every element of $\mathcal{X} K$, for every element of $L, L$, if $K \cap L=\varnothing$, then there exists an element of $C_{b}(L), f$, such that $f(K)=\{1\}$ and $f(L) \subset\{0\}$ and $0 \leq f \leq 1$. ®

If $\mathcal{L}$ satisfies condition $\otimes$, is separating and disjunctive, $T_{2}$, and strongly measure replete, then $T I_{M^{+} R(\sigma, L)}=\tau / M^{+} R(\sigma, L) \cdot$

Proof. Assume $\mathcal{L}$ satisfies condition $\otimes$, is separating and etc. 
$\alpha$ ) By Theorem 3.13, b), $\mathcal{T}$ is stronger that $\tau$.

B) Show $\tau /_{M^{*} R(\sigma, c)}$ is stronger than $T /_{M^{*} R(\sigma, c)^{*}}$

Consider any element of $M^{+} R(\sigma, L), \mu_{0}$, and any member of the subbase for the $\mathcal{T}_{M^{*} R(\sigma, L)}$-neighborhood system of $\mu_{0}, B\left(\mu_{0}, L^{\prime}, \varepsilon\right)$. (See the observation following the definition of $\mathcal{T}$.)

Since $L$ is strongly measure replete, $M^{+} R(\sigma, L) \subset M^{+} R(t, L)$. Consequently $\mu_{0} \in M^{+} R(t, L)$. Hence there exists an element of $\mathcal{X}, K$, such that $\left(\mu_{0}\right)_{*}\left(K^{\prime}\right)<\frac{2}{4}$. Consider any such $K$.

Since $\mathcal{L}$ is $T_{2}, \mathcal{X} \subset t L$.

Since $\mathcal{L}$ is strongly measure replete, it is measure replete. Consequently $\mu_{0} \in M^{+} R(\tau, L)$. Hence, since $L$ is separating and disjunctive, by ([4], Theorem 2.5), there exists an element of $M^{+} R(\tau, t L), \mu_{1}$, such that $\mu_{1} / q(\mathcal{l})=\mu_{0}$ and $\mu_{1}$ is unique. Now, note $\mu_{1}=\left(\mu_{0}\right)^{*}$ on $t \mathcal{L}$. Consequently $\mu_{1}=\left(\mu_{0}\right)^{*}$ on $\mathcal{X}$. Hence, since $\left(\mu_{0}\right)_{*}\left(K^{\prime}\right)+\left(\mu_{0}\right)^{*}(K)=\mu_{0}(X),\left(\mu_{0}\right)_{*}\left(K^{\prime}\right)=\mu_{1}\left(K^{\prime}\right)$. Consequently $\mu_{1}\left(K^{\prime}\right)<\frac{8}{4}$. Since $\mu_{0} \in M^{+} R(L)$, there exists an element of $\mathcal{L}, L_{1}$, such that $L_{1} \subset L^{\prime}$ and $\mu_{0}\left(L^{\prime}-L_{1}\right)<\frac{\varepsilon}{4}$. Consider any such $L_{1}$. Then consider $K \cap L_{1}$. Note $K \cap L_{1} \in \mathcal{X}$. Set $K \cap L_{1}=K_{1}$. Note $\mu_{1}\left(L^{\prime}-K_{1}\right) \leq \mu_{1}\left(K^{\prime}\right)+\mu_{0}\left(L^{\prime}-L_{1}\right)<\frac{i}{4}+\frac{i}{4}-\frac{8}{2}$.

Since $K_{1}=K \cap L_{1}$ and $L_{1} \subset L^{\prime}, K_{1} \subset L^{\prime}$. Hence, since $\mathcal{L}$ satisfies condition $\otimes$, there exists an element of $C_{b}(L), f$, such that $f\left(K_{1}\right)=\{1\}$ and $f(L) \subset\{0\}$ and $0 \leq f \leq 1$. Consider any such $f$.

Now, consider the following member of the subbase for the $\tau /{ }_{M^{+} R(\sigma, L)}$-neighborhood system of $\mu_{0}: N\left(\mu_{0} ; f, 1 ; \frac{\varepsilon}{2}\right)$. Show $N\left(\mu_{0} ; f, 1 ; \frac{\varepsilon}{2}\right) \subset B\left(\mu_{0}, L^{\prime}, \varepsilon\right)$. Consider any element of $N\left(\mu_{0} ; f, 1 ; \frac{2}{2}\right), v$. Note $\mu_{0}\left(L^{\prime}\right)=\mu_{1}\left(L^{\prime}\right)<\mu_{1}\left(K_{1}\right)+\frac{e}{2}=\int_{K_{1}} f d \mu_{1}+\frac{e}{2} \leq \int f d \mu_{1}+\frac{e}{2}=\int f d \mu_{0}+\frac{e}{2}<\left(\int f d v+\frac{e}{2}\right)+\frac{e}{2}=\iint_{L} f d v+\int_{L^{\prime}} f d v+\varepsilon=\int_{L^{\prime}} f d v+\varepsilon \leq v\left(L^{\prime}\right)+\varepsilon$. Further, note since $v \varepsilon N\left(\mu_{0} ; f, 1 ; \frac{\varepsilon}{2}\right),\left|v(X)-\mu_{0}(X)\right|<\varepsilon$. Consequently $v \in B\left(\mu_{0}, L^{\prime}, \varepsilon\right)$. Thus $N\left(\mu_{0} ; f, 1 ; \frac{i}{2}\right)$ $\subset B\left(\mu_{0}, L^{\prime}, \varepsilon\right)$. Hence $\tau /_{M^{+} R(\sigma, L)}$ is stronger than $\mathcal{T}_{M^{*} R(\sigma, L)}$.

Consequently $T /_{M^{+} R(\sigma, \mathcal{L})}=\tau /{ }_{M^{+} R(0, \mathcal{L})}$.

Example. Consider any topological space $X$ such that $X$ is locally compact, Lindelöf, and $T_{2}$, and let $L=\mathcal{F}$. Then, by ([4], p. 1516, Application 2$), \mathcal{F}$ is strongly measure replete. Consequently $T / M^{+} R(\sigma, \mathcal{F})=\left.w^{*}\right|_{M^{*} R\left(\sigma, T^{*}\right.}$

\section{Section 4.}

In this section conditions are obtained under which certain subsets of $M^{+} R(L)$ are sequentially closed in the weak topology.

Theorem 4.1. If $L$ is countably paracompact, $\delta$ and normal, then $M^{+} R(\sigma, L)$ is sequentially closed (in $M^{+} R(L)$ ).

Proof. Assume $\mathcal{L}$ is countably paracompact, $\delta$ and normal. Consider any sequence in $M^{+} R(\sigma, \mathcal{L}),\left\langle\mu_{n}\right\rangle$, and any element of $M^{+} R(L), v$, such that $\lim \mu_{n}=v$ in $w^{*}$. Show $v \in M^{+} R(\sigma, L)$.

a) For every $n$, consider $\mu_{n} / g(z(\mathcal{L}))$ and denote it by $\lambda_{n}^{0}$.

Observation. Consider any element of $M^{+} R(\sigma, \mathcal{L}), \lambda$. Now, consider $\lambda_{\Omega(z(\mathcal{L}))}$ and denote it by $\lambda^{0}$. Since $\lambda \in M^{+}(\sigma, L), \lambda^{0} \in M^{+}(\sigma, Z(L))$. Hence, since $Z(L)$ is complement generated, $\lambda^{0} \in M^{+} R(Z(L))$. Consequently $\lambda^{0} \in M^{+} R(\sigma, Z(L))$.

By the above observation, for every $n, \mu_{n}^{0} \in M^{+} R(\sigma, Z(L))$.

b) Consider $v / q(z(s))$ and denote it by $v^{0}$.

Observation. Consider any element of $M^{+} R(L), \lambda$. Now, consider $\lambda_{\wedge(z(L))}$ and denote it by $\lambda^{0}$. Since $L$ is $\delta$ and normal, $Z(L)$ semiseparates $L$. Hence $\lambda^{0} \in M^{+} R(Z(L)$ ).

By the above observation, $v^{0} \in M^{+} R(Z(L))$.

Since $\lim _{n} \mu_{n}=v$ in $w^{*}$, for every element of $C_{b}(\mathcal{L}), f, \lim _{n} \int f d \mu_{n}=\int f d v$. 
Observation. Consider any element of $M^{+}(L), \lambda$. Now, consider $\lambda /_{\Omega(z(L))}$ and denote it by $\lambda^{0}$. Further, consider any element of $C_{b}(L), f$. Note $\int f d \lambda=\int f d \lambda^{0}$.

By the above observation, for every element of $C_{b}(\mathcal{L}), f$, for every $n, \int f d \mu_{n}-\int f d \mu_{n}^{0}$ and $\int f d v=\int f d v^{0}$. Consequently $\lim _{n} \int f d \mu_{n}^{0}=\int f d v^{0}$. Then, by ([3], p. 209, Theorem 3), $v^{0} \in M^{+} R(\sigma, Z(L))$.

Since $L$ is $\delta$ and normal, $Z(L)$ separates $L$. Hence, since $\mathcal{L}$ is countably paracompact, $\mathcal{L}$ is $Z(L)$-countably paracompact. Consequently $v \in M^{+} R(\sigma, L)$.

Examples. (1). Consider any topological space $X$ such that $X$ is countably paracompact and normal, and let $\mathcal{L}=\mathcal{F}$. Then $M^{+} R(\sigma, \mathcal{F})$ is sequentially closed (in $M^{+} R(\mathcal{F})$ ).

(2). Consider any topological space $X$ such that $X$ is $T_{3 \frac{1}{2}}$ and countably bounded, and let $\mathcal{L}=\mathcal{F}$. Then by ([16], p. 268, Lemma 10$), Z$ semiseparates $\mathcal{F}$. Also, note the condition " $X$ is countably bounded" is equivalent to "F is $Z$ countably bounded." Consequently $M^{+} R(\sigma, \mathcal{F})$ is sequentially closed (in $M^{+} R(\mathcal{F})$ ).

The following Lemma will be needed on several occasions.

Lemma 4.2. If $\mathcal{L}$ is $\delta$ and $L$ semiseparates $t L$, then for every net in $M^{+} R(0, \mathcal{L}),\left\langle\mu_{a}\right\rangle$, for every element of $M^{+} R(\sigma, L), v$, if $\lim _{\alpha} \mu_{\alpha}=v$ in $T$, then for every element of $t L, F, \varlimsup_{\alpha} \mu_{\alpha}^{*}(F) \leq v^{*}(F)$, (equivalently, for every element of $\left.(t L)^{\prime}, U, \lim _{\alpha}\left(\mu_{\alpha}\right) .(U) \geq v_{*}(U)\right)$.

Proof. Assume $\mathcal{L}$ is $\delta$ and $\mathcal{L}$ semiseparates $t \mathcal{L}$. Consider any net in $M^{+} R(\sigma, L),\left\langle\mu_{a}\right\rangle$, and any element of $M^{+} R(\sigma, L), v$, such that $\lim _{\alpha} \mu_{\alpha}=v$ in $T$. Consider any element of $t L, F$.

a) Show $v^{*}(F)=\inf \{v(L) / L \in \mathcal{L}$ and $L \supset F\}$. Since $L$ is $\delta$ and $v \in M^{+} R(L), v^{*}(F)-\inf \left\{v\left(L^{\prime}\right) / L \in \mathcal{L}\right.$ and $\left.L^{\prime} \supset F\right\}$. Consider any element of $\mathcal{L}, L$, such that $L^{\prime} \supset F$. Then $F \cap L=\varnothing$. Hence, since $\mathcal{L}$ semiseparates $t \mathcal{L}$, there exists an element of $L, \hat{L}$, such that $\hat{L} \supset F$ and $\hat{L} \cap L=\varnothing$. Consider any such $\hat{L}$. Then $F \subset \hat{L} \subset L^{\prime}$. Hence $\inf \{v(\tilde{L}) / \tilde{L} \in \mathcal{L}$ and $\tilde{L} \supset F\} \leq v(\hat{L}) \leq v\left(L^{\prime}\right)$. Hence $\inf \{v(\tilde{L}) / L \in \mathcal{L}$ and $\tilde{L} \supset F\} \leq \inf \left\{v\left(L^{\prime}\right) / L \in \mathcal{L}\right.$ and $\left.L^{\prime} \supset F\right\}$. Consequently $\inf \{v(\tilde{L}) / \tilde{L} \in \mathcal{L}$ and $\tilde{L} \supset F\} \leq v^{\circ}(F)$. Further, note $v^{\circ}(F) \leq \inf \{v(\tilde{L}) / L \in L$ and $\tilde{L} \supset F\}$. Consequently $v^{*}(F)=\inf \{v(\tilde{L}) / \tilde{L} \in \mathcal{L}$ and $\tilde{L} \supset F\}$.

b) To show $\varlimsup \mu_{a}^{*}(F) \leq v^{*}(F)$, assume the contrary, namely, assume $\varlimsup \overline{\lim } \mu_{a}^{*}(F)>v^{*}(F)$. Then, by the result of part a), $\varlimsup_{\alpha} \mu_{a}^{*}(F)>\inf \{v(L) / L \in \mathcal{L}$ and $L \supset F\}$. Hence there exists an element of $\mathcal{L}, L$, such that $L \supset F$ and $\varlimsup_{\alpha} \mu_{\alpha}^{*}(F)>v(L)$. Consider any such $L$. Then, since $\lim _{\alpha} \mu_{\alpha}=v$ in $T, \varlimsup_{\alpha} \mu_{\alpha}(L) \leq v(L)$. Consequently $\varlimsup_{\alpha} \mu_{\alpha}^{*}(F)>\varlimsup_{\alpha} \mu_{\alpha}(L)$. Further, note for every $\alpha$, since $L \supset F, \mu_{\alpha}(L) \geq \mu_{\alpha}^{*}(F)$. Hence $\varlimsup_{\alpha}$ $\mu_{\alpha}(L) \geq \varlimsup_{a} \mu_{\alpha}^{*}(F)$. Thus a contradiction has been reached. Consequently $\varlimsup_{\alpha} \mu_{a}^{*}(F) \leq v^{*}(F)$.

Theorem 4.3. Consider $\left\{\mu_{x} ; x \in X\right\}$ and denote it by $D(L)$. If $(\mathcal{L}$ is $\delta$ and $\mathcal{L}$ semiseparates $t \mathcal{L}$, or for every subset of $X, S$, if $S$ is countable, then $S \in \mathcal{L}$ ), and $\mathcal{L}$ is separating, normal, and disjunctive, then $D(L)$ is sequentially closed in $M^{+} R(0, L)$.

Outline of a proof. Assume ( $L$ is $\delta$ and $\mathcal{L}$ semiseparates $t \mathcal{L}$, or for every subset of $X, S$, if $S$ is countable, then $S \in \mathcal{L}$ ), and etc. Since $\mathcal{L}$ is disjunctive, $D(\mathcal{L}) \subset I R(\sigma, \mathcal{L})$. Consider any sequence in $D(\mathcal{L}),\left\langle\mu_{x_{n}}\right\rangle$, and any element of $M^{+} R(\sigma, L), v$, such that $\lim \mu_{x_{n}}=v$ in $\mathcal{T}$. Since $\mathcal{L}$ is normal, by Corollary 3.7, $\operatorname{IR}(\mathcal{L})$ is closed. Consequently $v \in \mathbb{R}(\sigma, L)$. Consider $\left\{x_{1}, x_{2}, \ldots\right\}$ and denote it by $A$. Assume for any two values of $n, i, j$, if $i \neq j$, then $x_{i} \neq x_{j}$.

Case I. There exists an element of $X$, $y$, such that for every element of $L, L$, if $y \in L^{\prime}$, then $A \cap\left(L^{\prime}-\{y\}\right) \neq \varnothing$. Consider any such $y$. Then $v=\mu_{y}$. Consequently $v \in I R(\sigma, L)$.

Case II. For every element of $X, y$, there exists an element of $L, L$, such that $y \in L^{\prime}$ and 
$A \cap\left(L^{\prime}-\{y\}\right)=\varnothing$. Then $\left.\alpha\right) v^{*}(A)=0$ and $\left.\left.\beta\right) A \in t L . \gamma\right)$ Since $A \in t L$ and $\lim _{n} \mu_{x_{n}}-v$ in $\tau$ and $(\mathcal{L}$ is $\delta$ and $\mathcal{L}$ semiseparates $t \mathcal{L}$, or for every subset of $X, S$, if $S$ is countable, then $S \in \mathcal{L}$ ), by Lemma 4.2, $\varlimsup_{n} \mu_{x_{n}^{*}}^{*}(A) \leq v^{*}(A)$. Note $\varlimsup_{n} \mu_{x_{n}}^{*}(A)=1$. Consequently $\nu^{*}(A)=1$. Thus a contradiction has been reached. Consequently Case II does not occur.

Examples. Consider any topological space $X$ such that $X$ is normal and $T_{1}$.

(1). Let $\mathcal{L}=\mathcal{F}$. Since $(\mathcal{F}$ is $\delta$ and $\mathcal{F}$ semiseparates $t \mathcal{F}(=\mathcal{F})$ ), and $\mathcal{F}$ is separating, normal, and disjunctive, $D(\mathcal{F})$ is sequentially closed in $M^{+}(\sigma, \mathcal{F})$.

(2). Let $\mathcal{L}=Z$. Since $(Z$ is $\delta$ and $Z$ semiseparates $t Z(=\mathcal{F})$ ), and $Z$ is separating, normal, and disjunctive, $D(Z)$ is sequentially closed in $M^{+} R(\sigma, Z)$. ([20]).

The following three Lemmas will be needed in obtaining conditions under which $M^{+} R(\tau, L)$ is sequentially closed in $M^{+} R(\sigma, L)$.

Lemma 4.4. For every element of $M^{+} R(\sigma, L), v$, if there exists a subset of $X, X_{0}$, such that $X_{0} \cap \mathcal{L}$ is Lindelöf and $v^{*}\left(X_{0}\right)=v(X)$, then $v \in M^{+} R(\tau, L)$.

Proof. Consider any element of $M^{+} R(\sigma, \mathcal{L}), v$. Assume there exists a subset of $X, X_{0}$, such that $X_{0} \cap \mathcal{L}$ is Lindelöf and $v^{*}\left(X_{0}\right)=v(X)$. Consider any such $X_{0}$. To show $v \in M^{+} R(\tau, L)$, consider any net in $L,\left\langle L_{\alpha}\right\rangle$, such that $\left\langle L_{\alpha}\right\rangle$ is decreasing and $\lim _{\alpha} L_{\alpha}=\varnothing$ and show $\lim _{\alpha} v\left(L_{\alpha}\right)=0$.

a) Since $v^{*}\left(X_{0}\right)=v(X), X_{0}$ is $v$-thick. (See [12], pp. 74, 75.) Recall $A\left(X_{0} \cap L\right)-X_{0} \cap A(L)$ and consider the function $v_{0}$ which is such that $D_{v_{0}}=\mathfrak{A}\left(X_{0} \cap \mathcal{L}\right)$ and for every element of $\mathfrak{A}\left(X_{0} \cap \mathcal{L}\right)$, $X_{0} \cap A, v_{0}\left(X_{0} \cap A\right)=v(A)$. Recall $v_{0}$ is called the projection of $v$ to $X_{0}$. Note for every $\alpha, v\left(L_{\alpha}\right)=v_{0}\left(X_{0} \cap L_{\alpha}\right)$. Since $\left\langle L_{\alpha}\right\rangle$ is decreasing, $\left\langle X \cap L_{\alpha}\right\rangle$ is decreasing and, since $\lim _{\alpha} L_{\alpha}=\varnothing, \lim \left(X \cap L_{\alpha}\right)-\varnothing$. Further, note $\lim v\left(L_{\alpha}\right)=\lim v_{0}\left(X_{0} \cap L_{\alpha}\right)$.

B) Note $v_{0} \in M^{+} R\left(\sigma, X_{0} \cap \mathcal{L}\right)$. Since $X_{0} \cap \mathcal{L}$ is Lindelof, $M^{+} R\left(\sigma, X_{0} \cap \mathcal{L}\right) \subset M^{+} R\left(\tau, X_{0} \cap \mathcal{L}\right)$. Consequently $v_{0} \in M^{+} R\left(\tau, X_{0} \cap L\right)$.

y) Consequently $\lim v_{0}\left(X_{0} \cap L_{\alpha}\right)=0$. Consequently $\lim v\left(L_{\alpha}\right)=0$, and $v \in M^{+} R(\tau, L)$.

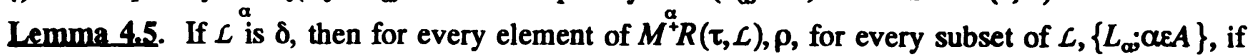
$\left\{L_{\alpha} ; \alpha \in A\right\}$ is a filter base, then $\rho^{*}\left(\bigcap_{\alpha} L_{\alpha}\right)=\underset{\alpha}{\inf } \rho\left(L_{\alpha}\right)$. ([18].) (For the special case of $\mathcal{L}=Z$ in a $T_{3 \frac{1}{2}}$ space, see [20].)

Lemma 4.6. If a topological space satisfies the countable chain condition and is paracompact, then it is Lindelöf. (Well-known.)

Theorem 4.7. If $L$ is $\delta$ and $L$ semiseparates $t L, t L$ is paracompact, and $L$ is separating and disjunctive, then $M^{+} R(\tau, L)$ is sequentially closed in $M^{+} R(\sigma, L)$. (For related theorems with $L=Z$, see [17].)

Proof. Assume $\mathcal{L}$ is $\delta$ and $\mathcal{L}$ semiseparates $t \mathcal{L}, t \mathcal{L}$ is paracompact, etc. Consider any sequence in $M^{+} R(\tau, L),\left\langle\mu_{n}\right\rangle$, and any element of $M^{+} R(\sigma, L), v$, such that $\lim \mu_{n}=v$ in $\mathcal{T}$. Show $v \in M^{+} R(\tau, L)$.

1. Show $\overline{\cup S\left(\mu_{n}\right)}$ is Lindelöf.

$\alpha)$ Show $\overline{U S\left(\mu_{n}\right)}$ satisfies the countable chain condition. Consider any subset of $(t L)^{\prime},\left\{0_{\alpha} ; \alpha \in A\right\}$, such that $\left\{\overline{U_{n} S\left(\mu_{n}\right)} \cap 0_{\alpha} ; \alpha \in A\right\}$ is disjoint and $A \neq \varnothing$ and for every $\alpha, \overline{U S\left(\mu_{n}\right)} \cap 0_{\alpha} \approx \varnothing$. Show $\left\{\overline{U S\left(\mu_{n}\right)} \cap 0_{\alpha} ; \alpha \in A\right\}$ is countable. For every $n$, consider $\left\{\alpha \in A / S\left(\mu_{n}\right) \cap 0_{\alpha} \neq \varnothing\right\}$ and denote it by $A_{n}$. Show $A=\bigcup_{n} A_{n}$. Consider any $\alpha$. Then $\bigcup_{n} S\left(\mu_{n}\right) \cap 0_{\alpha} \neq \varnothing$. Hence $\bigcup_{n} S\left(\mu_{n}\right) \cap 0_{\alpha} \neq \varnothing$. Hence there exists 
an $n$ such that $S\left(\mu_{n}\right) \cap 0_{\alpha} \neq \varnothing$. Consider any such $n$. Then $\alpha \in A_{n}$. Hence $\alpha \in \cup_{n} A_{n}$. Consequently $A=\cup_{n} A_{n}$. Note for every $n,\left\{S\left(\mu_{n}\right) \cap 0_{\alpha} ; \alpha \in A_{n}\right\}$ is disjoint and for every $\alpha$, if $\alpha \in A_{n}$, then $S\left(\mu_{n}\right) \cap 0_{\alpha} \neq \varnothing$; hence,

since $S\left(\mu_{n}\right)$ satisfies the countable chain condition (because $\mu_{n} \in M^{+} R(\tau, L)$ and $L$ is separating and disjunctive), $\left\{S\left(\mu_{n}\right) \cap 0_{\alpha} ; \alpha \in A_{n}\right\}$ is countable; hence $A_{n}$ is countable. Consequently $A$ is countable. Hence

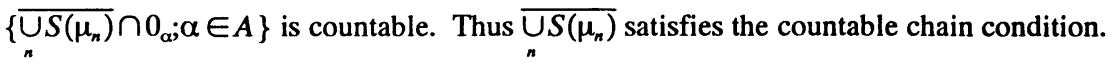

$\beta)$ Since $\overline{U S\left(\mu_{n}\right)} \in t L$ and $t L$ is paracompact, $\overline{\cup\left(\mu_{n}\right)}$ is paracompact. Hence, since $\overline{U S\left(\mu_{n}\right)}$ satisfies the countable chain condition, by Lemma 4.6, $\frac{n}{\cup\left(\mu_{n}\right)}$ is Lindelöf.

2. Show $v^{*}\left(\bigcup_{n} S\left(\mu_{n}\right)\right)=v(X)$. Since $\overline{\bigcup_{n} S\left(\mu_{n}\right)} \in t \mathcal{L}$ and $\lim _{n} \mu_{n}=v$ in $\mathcal{T}$ and $\mathcal{L}$ is $\delta$ and $\mathcal{L}$ semiseparates $t L$, by Lemma $4.2, \varlimsup_{n} \mu_{n}^{\cdot}\left(\bigcup_{k} S\left(\mu_{k}\right)\right) \leq v^{*}\left(\bigcup_{k}^{n} S\left(\mu_{k}\right)\right.$. Note for every $n, \mu_{n}^{*}\left(S\left(\mu_{n}\right)\right) \leq \mu_{n}^{\cdot}\left(\cup S\left(\mu_{k}\right)\right.$; since $\mu_{n} \in M^{+} R(\tau, L)$ and $L$ is $\delta$, by Lemma $4.5, \mu_{n}^{*}\left(S\left(\mu_{n}\right)\right)=\mu_{n}(X)$; consequently $\mu_{n}(X) \leq \mu_{n}^{*}\left(\bigcup_{k} S\left(\mu_{k}\right)\right)$. Hence $\varlimsup_{n} \mu_{n}(X) \leq \varlimsup_{n} \mu_{n}^{*}\left(\bigcup_{k} S\left(\mu_{k}\right)\right.$. Since $\lim _{n} \mu_{n}=v$ in $\mathcal{T}, \lim _{n} \mu_{n}(X)=v(X)$. Consequently $v(X) \leq \varlimsup_{n} \mu_{n}^{*}$ $\left(\bigcup_{k} S\left(\mu_{k}\right)\right.$. Hence $v^{*}\left(\bigcup_{n} S\left(\mu_{n}\right)\right)=v(X)$.

3. Consequently $\overline{\bigcup_{n} S\left(\mu_{n}\right)} \cap \mathcal{L}$ is Lindelöf and $v^{*}\left(\bigcup_{n} S\left(\mu_{n}\right)\right)=v(X)$. Hence, by Lemma 4.4, $v \in M^{+} R(\tau, L)$.

Thus $M^{+} R(\tau, L)$ is sequentially closed in $M^{+} R(\sigma, L)$.

Example. Consider any topological space $X$ such that $X$ is paracompact and $T_{1}$, and let $\mathcal{L}=\mathcal{F}$. Since $\mathcal{F}$ is $\delta$ and semiseparates $t \mathcal{F}(=\mathcal{F}), t \mathcal{F}$ is paracompact, and $\mathcal{F}$ is separating and disjunctive, $M^{+} R(\tau, \mathcal{F})$ is sequentially closed in $M^{+} R(\sigma, \mathcal{F})$. (This result is also true in normal metacompact spaces; see [17].)

\section{Section 5.}

In this section conditions are obtained under which tightness implies relative compactness in the weak topology and vice versa.

Theorem 5.1. If $L$ is $\delta$ and $\mathcal{L}$ semiseparates $t L$, and $L$ is separating, disjunctive, and normal, then for every subset of $M^{+} R(\sigma, L), A$, if $A$ is tight, then $A$ is $w^{*}$-relatively compact in $M^{+} R(\sigma, L)$.

Proof. Assume $\mathcal{L}$ is $\delta$ and $\mathcal{L}$ semiseparates $t \mathcal{L}$, and $\mathcal{L}$ is separating, disjunctive, and normal. Consider any subset of $M^{+} R(\sigma, L), A$, such that $A$ is tight. Then, by definition, $A$ is norm bounded. Hence there exists a positive number, $k$, such that for every element of $M R(L), \rho$, if $\rho \in A$, then $\|\rho\| \leq k$. Consider any such $k$. Now, consider $\{\rho \in M R(L)\|\rho\| \leq k\}$. Note $A \subset\{\rho \in M R(L)\|\rho\| \leq k\}$. Hence $\bar{A} \subset$ $\{\rho \in M R(L) \| \rho \rho \leq k\}$. The following fact is well-known: $\{\rho \in M R(L)\|\rho\| \leq k\}$ is compact. Hence, since the topology $w^{*}$ is $T_{2},\{\rho \in M R(L)\|\rho\| \leq k\}$ is closed. Consequently $\bar{A} \subset\{\rho \in M R(L)\|\rho\| \leq k\}$. Consequently $\bar{A}$ is compact. Therefore to show $A$ is $w^{*}$-relatively compact in $M^{+} R(\sigma, L)$, it suffices to show $\bar{A} \subset M^{+} R(\sigma, L)$.

Case $A=\varnothing$. Then $\bar{A} \subset M^{+} R(\sigma, L)$.

Case $A \approx \varnothing$. Since $L$ is $\delta$ and normal, by Proposition 3.9, $M^{+} R(L)$ is closed. Hence, since $A \subset M^{+} R(\sigma, L), \bar{A} \subset M^{+} R(L)$. Consider any element of $\bar{A}, v$. Then $v \in M^{+} R(L)$. Hence to show $v \in M^{+} R(\sigma, \mathcal{L})$, it suffices to show for any sequence in $\mathcal{L},\left\langle L_{n}\right\rangle$, if $\left\langle L_{n}\right\rangle$ is decreasing and $\lim L_{n}=\varnothing$, then $\lim _{n} v\left(L_{n}\right)=0$. Consider any sequence in $L,\left\langle L_{n}\right\rangle$, such that $\left\langle L_{n}\right\rangle$ is decreasing and $\lim _{n} L_{n}=\varnothing$. Now, consider any positive number, $\varepsilon$. Since $A$ is tight, by definition, there exists an element of $\mathcal{K} K$, such that for every element of $A, \rho_{*}\left(K^{\prime}\right)<\varepsilon$. Consider any such $K$. Since $v \in \bar{A}$, there exists a net in $A$, $\left\langle\mu_{a}\right\rangle$, such that 
$\lim \mu_{\alpha}=\nu$. Consider any such $\left\langle\mu_{\alpha}\right\rangle$. Note for every $\alpha,\left(\mu_{\alpha}\right)_{\bullet}\left(K^{\prime}\right)<\varepsilon$. Since $\mathcal{L}$ is separating, disjunctive, and normal, $\mathcal{X} \subset t L$. Consequently $K \in t L$. Consequently $K \in t L$ and $\lim \mu_{\alpha}-\nu$, and $\mathcal{L}$ is $\delta$ and $L$ semiseparates $t \mathcal{~}$. Hence, by Lemma $4.2, v_{*}\left(K^{\prime}\right) \leq \underline{\lim }\left(\mu_{a}\right) .\left(K^{\prime}\right)$. Consequently $v_{*}^{\alpha}\left(K^{\prime}\right) \leq \varepsilon$. Since $\left\langle L_{n}\right\rangle$ is decreasing and $\lim _{n} L_{n}=\varnothing, \cap_{n} L_{n}=\varnothing$. Hence $K \cap\left(\cap_{n} L_{n}\right)=\varnothing$. Hence, since $\left\langle L_{n}\right\rangle$ is decreasing and $K \in \mathcal{X}$ there exists a value of $n, n_{0}$, such that for every $n$, if $n \geq n_{0}$, then $K \cap L_{n}=\varnothing$; equivalently $L_{n} \subset K^{\prime}$. Consider any such $n_{0}$. Then for every $n$, if $n \geq n_{0}$, then $v\left(L_{n}\right) \leq v \cdot\left(K^{\prime}\right) \leq \varepsilon$. Hence $\lim _{n} v\left(L_{n}\right)=0$. Consequently $v \in M^{+} R(\sigma, L)$. Hence $\bar{A} \subset M^{+} R(\sigma, L)$.

Consequently $A$ is $w^{*}$-relatively compact in $M^{+} R(\sigma, L)$.

Example. Consider any topological space $X$ such that $X$ is $T_{1}$ and normal, and let $L-\mathcal{F}$. Since $\mathcal{F}$ is $\delta$ and $\mathcal{F}$ semiseparates $t \mathcal{F}(=\mathcal{F})$, and $\mathcal{F}$ is separating, disjunctive, and normal, for every subset of $M^{+} R(\sigma, \mathcal{F})$, $A$, if $A$ is tight, then $A$ is $w^{\circ}$-relatively compact in $M^{+} R(\sigma, \mathcal{F})$.

The following theorem also gives conditions under which tightness implies relative compactness.

Theorem 5.2. If $\mathcal{L}$ is countably paracompact, separating and disjunctive, $\delta$ and normal, then for every subset of $M^{+} R(\sigma, L), A$, if $A$ is tight, then $A$ is $w^{*}$-relatively compact in $M^{+} R(\sigma, L)$. (Proof omitted.)

Examples. (1). Consider any topological space $X$ such that $X$ is countably paracompact, $T_{1}$, and normal, and let $\mathcal{L}=\mathcal{F}$. Then for every subset of $M^{+} R(\sigma, \mathcal{F}), A$, if $A$ is tight, then $A$ is $w^{*}$-relatively compact in $M^{+} R(\sigma, \mathcal{L})$.

(2). Consider any topological space $X$ such that $X$ is $T_{3 \frac{1}{2}}$, and let $L=Z$. Then for every subset of $M^{+} R(\sigma, Z), A$, if $A$ is tight, then $A$ is $w^{*}$-relatively compact in $M^{+} R(\sigma, Z)$. ([20], p. 205, Corollary III.)

(3). Consider any topological space $X$ such that $X$ is $T_{1}$, and let $L-B$. Then for every subset of $M^{+}(\sigma, \mathcal{B}), A$, if $A$ is tight, then $A$ is $w^{*}$-relatively compact in $M^{+}(\sigma, B)$.

The following theorem gives conditions under which relative compactness implies tightness.

Theorem 5.3. Consider the condition: For every element of $\mathcal{X} K$, there exist an element of $L, L$, and an element of $\mathcal{X} \tilde{K}$, such that $K \subset L^{\prime} \subset \tilde{K}$. C

If $L$ is separating and disjunctive, satisfies condition $\mathcal{C}$, is strongly measure replete, $\delta$ and normal, then for every subset of $M^{+} R(\sigma, L), A$, if $A$ is $w^{*}$-compact, then $A$ is tight.

Proof. Assume $L$ is separating and disjunctive, satisfies etc. Consider any subset of $M^{+} R(\sigma, L), A$, such that $A$ is $w^{*}$-compact. Assume $A \neq \varnothing$.

$\alpha$ ) Show $A$ is norm bounded. Note for every element of $A, \mu,\|\mu\|=\mu(X)-\int 1 d \mu$. Consider the element of $C_{b}(\mathcal{L}), f$, which is such that $f=1$. Then, by definition (see p. 3 ), for every element of $M R(L), \mu, \hat{f}(\mu)-\int f d \mu$. Since $L$ is $\delta$ and normal, $\hat{f}$ is $w^{*}$-continuous. Hence, since $A$ is $w^{*}$-compact, $\hat{f}(A)$ is bounded. Consequently $A$ is norm bounded.

$\beta)$ Show for every positive number, $\varepsilon$, there exists an element of $\mathcal{K} K$, such that for every element of $A, \mu, \mu_{0}(X-K)<\varepsilon$. Consider any positive number $\varepsilon$. Now, consider any element of $A, \mu$.

Since $\mathcal{L}$ is strongly measure replete, $\mu \in M^{+} R(t, L)$. (See Theorem 3.15.) Hence there exists an element of $\mathcal{X} K_{\mu}$ such that $\mu_{0}\left(X-K_{\mu}\right)<\varepsilon$. Consider any such $K_{\mu}$.

Since $\mathcal{L}$ satisfies condition $\mathbb{O}$, there exist an element of $\mathcal{L}, L_{\mu}$ and an element of $\mathcal{X} \mathscr{K}_{\mu}$ such that $K_{\mu} \subset L_{\mu}{ }^{\prime} \subset \tilde{K}_{\mu}$. Consider any such $L_{\mu} \tilde{K}_{\mu}$.

Since $L$ is separating, disjunctive, and normal, $\mathcal{X} \subset t \mathcal{L}$.

Since $\mathcal{L}$ is strongly measure replete, it is measure replete. Consequently $\mu \in M^{+} R(\tau, L)$. Hence, since $\mathcal{L}$ is separating and disjunctive, by $([4]$, Theorem 2.5$)$, there exists an element of $M^{+} R(\tau, t \mathcal{L}), \mu_{1}$, such that $\mu_{1} / q(\mathcal{G})=\mu$ and $\mu_{1}$ is unique. Moreover, $\mu_{1}=\mu^{*}$ on $t \mathcal{~}$. Consequently $\mu_{1}=\mu_{0}$ on $X$.

Then $\mu\left(L_{\mu}\right)=\mu\left(X-L_{\mu}{ }^{\prime}\right)=\mu_{1}\left(X-L_{\mu}{ }^{\prime}\right) \leq \mu_{1}\left(X-K_{\mu}\right)=\mu_{0}\left(X-K_{m}\right)<\varepsilon$. 
Observation. Consider any element of $L, L$. Since $L$ is $\delta$ and normal, for every element of $C_{b}(L), f, \hat{f}$ is $w^{*}$-continuous. Now, consider the function $\psi$ determined by $\psi=\inf \left\{\hat{f} / f \in C_{B}(L)\right.$ and $f \geq \kappa_{L}$. By ([9], p. 85, Corollary 10.4, (b)), $\psi$ is $w^{*}$-upper-semicontinuous. Hence $\{v \in M R(L) / \psi(v)<\varepsilon\}$ is $w^{*}$-open. Note for every element of $M R(L), v, \psi(v)=\inf \left\{\hat{f}(v) / f \in C_{b}(L)\right.$ and $\left.f \geq \kappa_{L}\right\}$; further, note if $v \geq 0$, then, by Alexandroff's Representation Theorem, $\inf \left\{\hat{f}(v) / f \in C_{b}(L)\right.$ and $\left.f \geq \kappa_{L}\right\}=v(L)$. Consequently $\left\{v \in M^{+} R(L) / v(L)<\varepsilon\right\}$ is $w^{*}$-open.

By this observation, $\left\{v \in M^{+} R(\sigma, L) / v\left(L_{\mu}\right)<\varepsilon\right\}$ is $w^{*}$-open. Since $\mu\left(L_{\mu}\right)<\varepsilon, \mu \in\left\{v \in M^{+}\right.$ $\left.R(\sigma, L) / \nu\left(L_{\mu}\right)<\varepsilon\right\}$. Denote this set by $W_{\mu}$.

Note $\left\{W_{\mu}: \mu \in A\right\}$ is an open cover of $A$. Hence, since $A$ is compact, there exists a subcover of $A, \mathcal{S}$, such that $S$ is finite, and nonempty (since $A$ is nonempty). Consider any such $S$ and denote it by $\left\{W_{\mu_{n}} ; n=1, \ldots, C\right\}$. Also, consider $\cup\left\{\tilde{K}_{\mu_{n}} ; n=1, \ldots, C\right\}$. Note $\cup\left\{\tilde{K}_{\mu_{n}} ; n=1, \ldots, l\right\} \in \mathcal{X}$. Denote this set by $\tilde{R}$.

Now, consider any element of $A, \mu$. Since $A \subset \cup\left\{W_{\mu_{x}} ; n=1, \ldots, \ell\right\}$, there exists a value of $n, n_{0}$, such that $\mu \in W_{\mu_{x_{0}}}$. Consider any $\operatorname{such} n_{0}$. Then $\hat{K} \in \mathcal{X}$ and $\mu_{0}(X-\hat{K})=\mu_{1}(X-\hat{K}) \leq \mu_{1}\left(X-\tilde{K}_{\mu_{n_{0}}}\right) \leq \mu_{1}\left(X-L_{\mu_{n_{0}}}{ }^{\prime}\right)=$ $\mu\left(X-L_{\mu_{n_{0}}}\right)=\mu\left(L_{\mu_{n_{0}}}\right)<\varepsilon$.

$\gamma$ ) Consequently $A$ is tight.

Remark. This theorem is a mild generalization of a result of Varadarajan ([20], p. 205, Theorem 29) and implies readily the Prohorov theorem.

Examples. (1). Consider any topological space $X$ such that $X$ is $T_{1}$, locally compact, normal, and $\mathcal{F}$ is strongly measure replete, and let $\mathcal{L}=\mathcal{F}$. Then for every subset of $M^{+} R(\sigma, \mathcal{F}), A$, if $A$ is $w^{\circ}$ compact, then $A$ is tight.

Remarks. a) All conditions are satisfied, if $X$ is locally compact, $T_{2}$, and Lindelöf. (See [4], p. 1516, Application 2.)

b) All conditions are satisfied, if $X$ is locally compact, $T_{2}$, and paracompact and separable. (See [4], p. 1516, Application 2')

(2). Consider any topological space $X$ such that $X$ is $T_{2}$, locally compact, and $Z$ is strongly measure replete, and let $L=Z$. Then for every subset of $M^{+} R(\sigma, Z), A$, if $A$ is $w^{*}$-compact, then $A$ is tight.

Remark Conditions for $Z$ to be strongly measure replete are found in [6], e.g., $X \in \sigma(W(Z))$.

The following theorem is a generalization of Theorem 5.3.

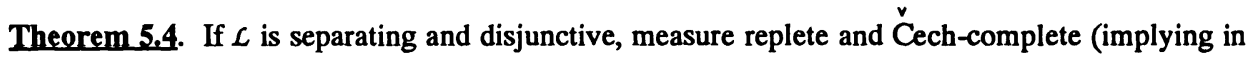
particular that $L$ is strongly measure replete) (see [4], p. 1516), then for every subset of $M^{+} R(\sigma, L), A$, if $A$ is $w^{*}$-compact, then $A$ is tight. (Proof omitted.)

Remark. In the concrete situation of Tychonoff spaces, with $\mathcal{L}=\mathcal{F}$, these results can be found in [13] and [19].

\section{Section 6.}

The following theorems describe a relationship between convergence in $\mathcal{T}$ and convergence in $\hat{\mathcal{T}}$, convergence in $\tilde{\mathcal{T}}$, or convergence in $T^{\prime}$. (For the definitions of $\hat{\mathcal{T}}, \tilde{\mathcal{T}}$, and $\tau^{\prime}$, see p. 4.)

[Denote the general element of $M^{+} R(L)$ by $\mu$. The following three functions, associated with $\mu$, occur in the theorems just mentioned, namely, $\hat{\mu}, \tilde{\mu}$, and $\mu^{\prime}$. For their definitions see ([4], p. 1501 and p. 1508).]

Theorem 6.1. For every net in $M^{+} R(L),\left\langle\mu_{\alpha}\right\rangle$, for every element of $M^{+} R(L), v, \lim _{\alpha} \mu_{\alpha}=v$ in $\mathcal{T}$ iff $\lim _{\alpha} \hat{\mu}_{\alpha}=\hat{v}$ in $\hat{\tau}$. (Proof omitted.)

Theorem 6.2. If $W(L)$ is $\delta$, then for every net in $M^{+} R(L),\left\langle\mu_{a}\right\rangle$, for every element of $M^{+} R(L), v, \lim _{\alpha} \mu_{\alpha}=v$ in $\tau$ iff $\lim _{\alpha} \tilde{\mu}_{\alpha}=\tilde{v}$ in $\tilde{\mathcal{T}}$. (Proof by using Lemma 4.2.) 
Corollary 6.3. If $L$ is countably compact, then for every net in $M^{+} R(L),\left\langle\mu_{a}\right\rangle$, for every element of $M^{+} R(\mathcal{L}), v, \lim _{\alpha} \mu_{\alpha}=v$ in $T$ iff $\lim _{\alpha} \tilde{\mu}_{\alpha}=\tilde{v}$ in $\tilde{T}$. (Proof omitted.)

Theorem 6.4. If $L$ is (separating), disjunctive, $\delta$, and normal, then for every net in $M^{+} R(L),\left\langle\mu_{a}\right\rangle$, for every element of $M^{+} R(\mathcal{L}), v, \lim _{\alpha} \mu_{\alpha}=v$ in $\mathcal{T}$ iff $\lim _{\alpha} \bar{\mu}_{\alpha}=\bar{v}$ in $\tilde{T}$.

Proof. Assume $\mathcal{L}$ is (separating), disjunctive, $\delta$, and normal. Consider any net in $M^{+} R(L),\left\langle\mu_{a}\right\rangle$, and any element of $M^{+} R(L), v$.

a) Assume $\lim _{\alpha} \mu_{\alpha}=v$ in $\tau$. Since $\mathcal{L}$ is normal, $t W(L)$ is normal. Consequently $t W(\mathcal{L})$ is $\delta$ and normal. Hence $\tilde{\mathcal{T}}$ is the weak topology. [See (Theorem 3.13, d)).] Therefore to show $\lim _{\alpha} \tilde{\mu}_{\alpha}=\bar{v}$ in $\tilde{\mathcal{T}}$, it suffices to show for every element of $C(t W(L)), g, \lim _{\alpha} \int g d \tilde{\mu}_{\alpha}=\int g d \bar{v}$.

Since $\mathcal{L}$ is (separating), disjunctive, $\delta$, and normal, the function ^ which maps the general element of $C_{b}(L), f$, onto the element of $C(t W(L)), \hat{f}$, which is such that $D_{f}=I R(L)$ and for every element of $\operatorname{IR}(L), \lambda, \hat{f}(\lambda)=\int f d \lambda$ is onto. ( ${ }^{\wedge}$ is also a vector-space isomorphism and norm preserving.)

Consequently to show $\lim _{\alpha} \tilde{\mu}_{\alpha}=\tilde{v}$ in $\tilde{\mathcal{T}}$, it suffices to show for every element of $C_{b}(L), f, \lim \int \hat{f} d \tilde{\mu}_{a}=\int \hat{f} d \bar{v}$.

For this purpose, consider the following

Lemma. If $L$ is (separating), disjunctive, $\delta$, and normal, then for every element of $M^{+} R(L), \lambda$, for every element of $C_{b}(L), f, \int \hat{f} d \hat{\lambda}=\int f d \lambda$. (Proof omitted.)

Consider any element of $C_{b}(L), f$. Note for every $\alpha$, by the Lemma, $\int \hat{f} d \bar{\mu}_{a}-\int f d \mu_{a}$. Since $\mathcal{L}$ is $\delta$ and normal, $\mathcal{T}$ is the weak topology. [See (Theorem 3.13, d)).] Therefore, since $\lim _{\alpha} \mu_{\alpha}=v$ in $\mathcal{T}$, $\lim _{\alpha} \int f d \mu_{\alpha}=\int f d v$. Consequently $\lim _{\alpha} \int \hat{f} d \tilde{\mu}_{\alpha}=\int f d v$. Again by the Lemma, $\int f d v=\int \hat{f} d \hat{v}^{\alpha}$. Consequently $\lim _{\alpha} \int \hat{f} d \tilde{\mu}_{\alpha}=\int \hat{f} d \tilde{v}$. Consequently $\lim _{\alpha} \tilde{\mu}_{\alpha}=\tilde{v}$ in $\tilde{\tau}$.

b) Assume $\lim \tilde{\mu}_{\alpha}=\tilde{v}$ in $\tilde{T}$. Then $\lim \mu_{\alpha}=v$ in $\mathcal{T}$. (Proof omitted.)

Theorem 6.5. For every net in $M^{\alpha} R(L),\left\langle\mu_{\alpha}\right\rangle$, for every element of $M^{+} R(L), v, \lim _{\alpha} \mu_{\alpha}=v$ in $\mathcal{T}$ iff $\lim \mu_{\alpha}^{\prime}=v^{\prime}$ in $\tau^{\prime}$. (Proof omitted.)

\section{ACKNOWLEDGMENT}

The author wishes to express his appreciation to Long Island University for partial support of the present work through a grant of released time from teaching duties.

\section{REFERENCES}

1. W. Adamski, An abstract approach to weak topologies in spaces of measures, Bull. Greek Math. Soc., 18 (1977), 28-68.

2. A. D. Alexandroff, Additive set-functions in abstract spaces, Mat. Sb. (N.S.) 9(51)(1941), 563-628.

3. Additive set-functions in abstract spaces, Mat. Sb. (N.S.) 13 (55) (1943), 169-238.

4. G. Bachman and P. Stratigos, Criteria for $\sigma$-smoothness, $\tau$-smoothness, and tightness of lattice regular measures, with applications, Can. J. Math., vol. XXXIII (1981), 1498-1525.

5. , On generallattice repleteness and completeness, IIl.J. Math., 27 (1983), 535-561.

6. On measure repleteness and support for lattice regular measures, Internat. J. Math. \& Math. Sci., Vol. 10, No. 4(1987) 707-724.

7. B. Banaschewski, Über nulldimensionale raume, Math. Nachr., 13 (1955), 129-140.

8. J. H. Blau, The space of measures on a given set, Fund. Math., 38 (1951), 23-34.

9. J. Dugundji, Topology (Allyn and Bacon, Boston, 1966),

10. L. Gillman and M. Jerison, Rings of continuous functions (Van Nostrand, Princeton, N.J. 1960).

11. P. Grassi, On subspaces of replete and measure replete spaces, Canad. Math. Bull. 27(1) (1984), 58-64. 
12. P. Halmos, Measure theory (Van Nostrand, Toronto, New York, London, 1950).

13. J. Hoffman-J $\phi r g e n s e n$, Weak compactness and tightness of subsets of $M(X)$, Math. Scand., 31 (1972), $127-150$.

14. G. Kallianpur, Topology of weak convergence of probability measures, J. Math. Mech., 10, 6(1951), 947-969.

15. J. Kelley, General topology (Van Nostrand, Toronto, New York, London, 1955).

16. J. Mack, Countable peracompactness and weak normality properties, Trans. A.M.S., 48 (1970), 265-271.

17. W. Moran, Measures on metacompact spaces, Proc. London Math. Soc., (3) 20 (1970), 507-529.

18. M. Szeto, Measure repleteness and mapping preservations, J. Indian Math. Soc., 43 (1979), 35-52.

19. F. Tops $\phi$ e, Compactness and tightness in a space of measures with the topology of weak convergence, Math. Scand., 34 (1974), 187-210.

20. V. Varadarajan, Measures on topological spaces, Amer. Math. Soc. Transl., Ser. 2, 48 (1965), 161-228.

21. H. Wallman, Lattices and topological spaces, Ann. of Math., 39 (1938), 112-126. 


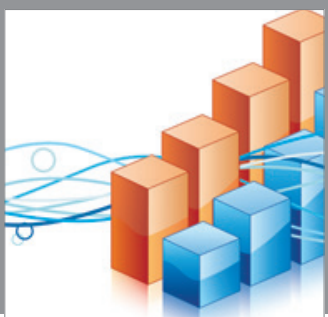

Advances in

Operations Research

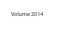

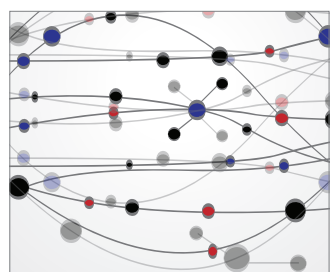

\section{The Scientific} World Journal
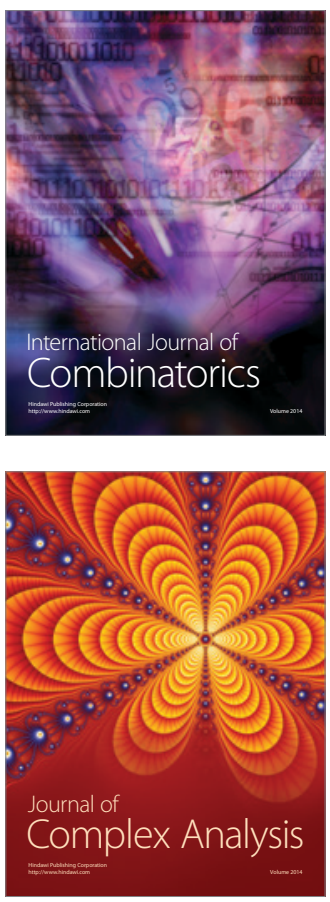

International Journal of

Mathematics and

Mathematical

Sciences
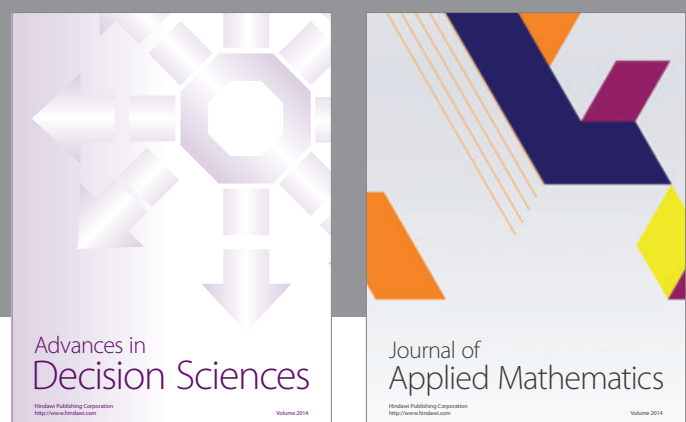

Journal of

Applied Mathematics
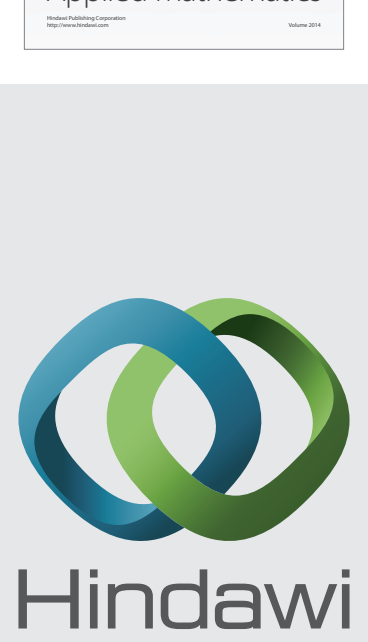

Submit your manuscripts at http://www.hindawi.com
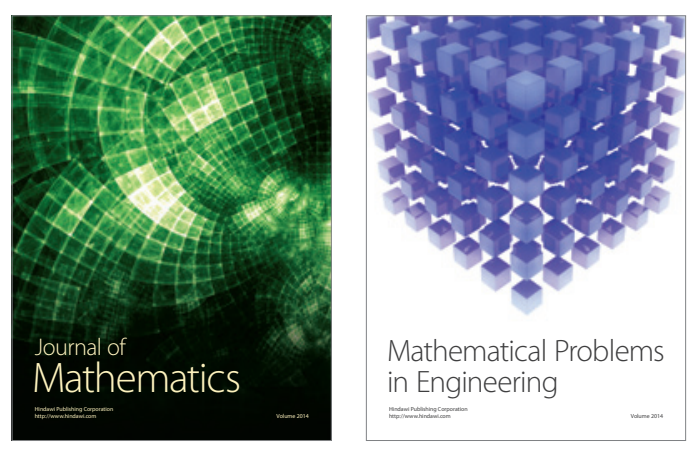

Mathematical Problems in Engineering
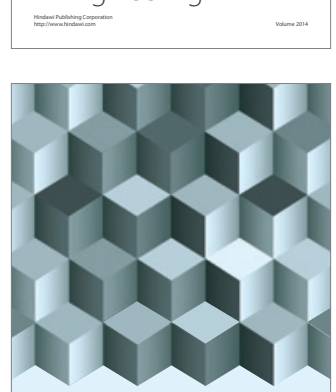

Journal of

Function Spaces
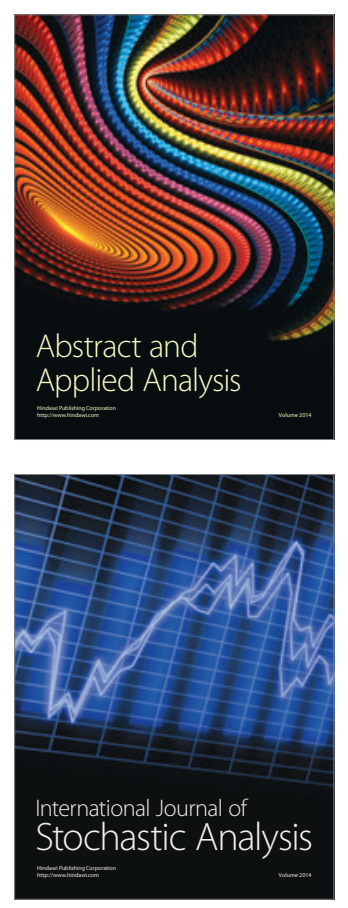

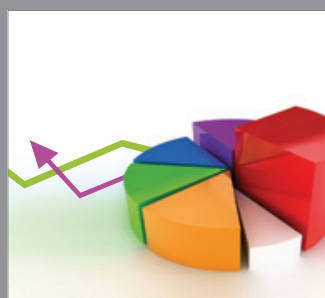

ournal of

Probability and Statistics

Promensencen
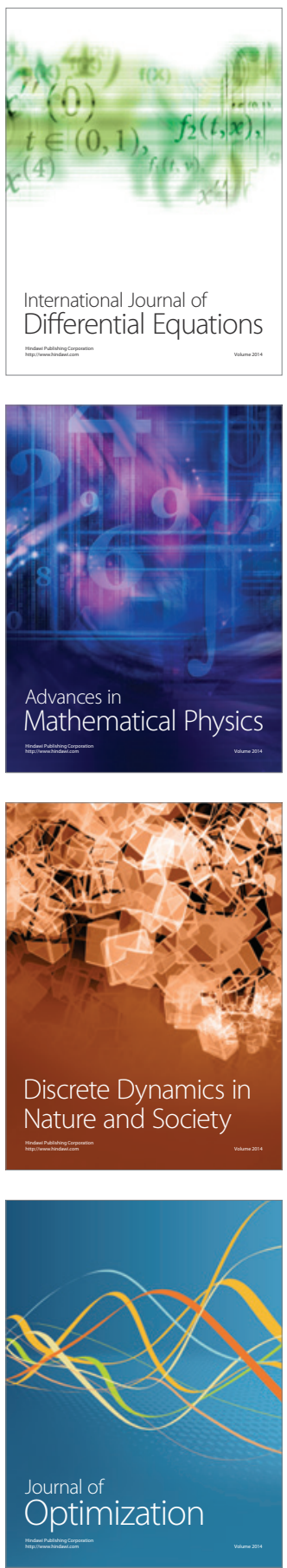